\title{
Fast and Robust Phase Unwrapping Algorithm for Electron Holography
}

\author{
M. A. Schofield and Y. Zhu \\ Department of Materials Science, Brookhaven National Laboratory, Upton, Long Island NY 11973
}

A major problem with off-axis electron holography and a range of other interferometric techniques that recover phase information is that the reconstructed phase is mathematically limited to the interval $(-\pi, \pi]$. In general, the true phase may range over a larger interval than $2 \pi$, so the phase that is obtained generally contains discontinuities. Unwrapping these discontinuities in the phase is, in principle, simple. One needs only add an appropriate integer multiple of $2 \pi$ to each pixel of the (experimental) wrapped phase. (For example, the simplest method is a pixel-by-pixel, pathdependent method that identifies phase jumps along the path and adds cumulative multiples of $2 \pi$ to subsequent phase values). In practice, a number of serious issues complicate or prevent effective phase unwrapping with, not only the above algorithm, but with more sophisticated algorithms as well. The presence of noise or vortex points (e.g., arising from regions of low fringe contrast in the hologram) is of primary concern for effective unwrapping of the reconstructed phase. Noise impedes the ability to reliably judge the existence of a phase jump, while vortices mean the unwrapped phase will be path-dependent. On the other hand, sophisticated algorithms that deal more effectively with these issues invariably suffer from lengthy computational time, demands for strong user input, or both. This also is problematic since the phase unwrapping problem is not just one of aesthetics: It is valuable to be able to quantitatively compare the experimental phase to, for example, model calculations without it being a major endeavor to simply unwrap the phase. It is, therefore, highly desirable to have an unwrapping algorithm that is reliable in the presence of noise and vortex points, and which does not demand strong user input or computation time.

We present here an algorithm to unwrap the phase that is largely immune to the above limitations ${ }^{1}$. We begin by writing the unwrapped phase, $\phi(\mathbf{r})$, in terms of the wrapped phase, $\phi_{\mathrm{w}}(\mathbf{r})$, so that $\phi(\mathbf{r})=\phi_{\mathrm{w}}(\mathbf{r})+2 \pi \mathrm{n}(\mathbf{r})$, where $\mathbf{r}$ refers to pixel position and $\mathrm{n}(\mathbf{r})$ is an integer field. Taking the 2-D Laplacian and solving for $\mathrm{n}(\mathbf{r})$, we obtain

$$
\mathrm{n}(\mathbf{r})=\frac{1}{2 \pi} \nabla_{\perp}^{-2}\left[\nabla_{\perp}^{2} \phi(\mathbf{r})-\nabla_{\perp}^{2} \phi_{\mathrm{w}}(\mathbf{r})\right]
$$

where $\nabla_{\perp}^{2}$ and $\nabla_{\perp}^{-2}$ are the forward and inverse 2-D Laplacian operators, respectively. Eqn [1] may readily be solved using the Fourier representation of the forward and inverse Laplacian operators ${ }^{2}$, provided we know how to calculate $\nabla_{\perp}^{2} \phi(\mathbf{r})$ with only knowledge of the wrapped phase, $\phi_{\mathrm{w}}(\mathbf{r})$. This can be done $\mathrm{e}^{3}$ by defining the complex quantity, $\mathrm{P}(\mathbf{r})=\mathrm{e}^{i \phi_{\mathrm{w}}(\mathbf{r})}=\mathrm{e}^{i[\phi(\mathbf{r})-2 \pi \mathrm{n}(\mathbf{r})]}=\mathrm{e}^{i \phi(\mathbf{r})}$, since $\mathrm{n}(\mathbf{r})$ is an integer, and recognizing that $\operatorname{Im}\left(\frac{1}{\mathrm{P}} \nabla_{\perp}^{2} \mathrm{P}\right)=\nabla_{\perp}^{2} \phi$, where $\operatorname{Im}(\cdots)$ denotes imaginary part. Since $\mathrm{P}=\cos \phi_{\mathrm{w}}+i \sin \phi_{\mathrm{w}}$, Eqn [1] may be solved, and the solution is unique once boundary conditions are imposed, along with the constraint that $\mathrm{n}(\mathbf{r})$ be a field of integers.

The constraint that $\mathrm{n}(\mathbf{r})$ be integers can be done in implementation by rounding the solution obtained from Eqn [1]. Applying Fourier techniques to calculate the forward and inverse Laplacians imposes periodic boundary conditions on the solution obtained for $\mathrm{n}(\mathbf{r})$, which isn't generally realistic. More realistic is that the gradient of $n(\mathbf{r})$ normal to the boundary vanishes. Except for the unfortunate situation where a phase jump occurs precisely along the boundary, this is a good approximation since $\mathrm{n}(\mathbf{r})$ are integers. The boundary condition that the normal gradient vanishes is easily implemented by symmeterizing the input wrapped phase in the algorithm. ${ }^{4}$ That is, a synthesized image of dimension $2 \mathrm{Nx} 2 \mathrm{~N}$ is created by mirror reflection of the original $\mathrm{NxN}$ image about its boundary. The solution, therefore, also contains this mirror symmetry and the $\mathrm{NxN}$ solution for $\mathrm{n}(\mathbf{r})$ 
is obtained by extracting the proper quadrant of the $2 \mathrm{Nx} 2 \mathrm{~N}$ solution. Although the solution obtained from Eqn [1] is, then, unique, it may not completely unwrap the phase since the boundary conditions are only approximated, and a rounding step is introduced to obtain $\mathrm{n}(\mathrm{r})$. We may, however, use the partially unwrapped phase as input to another iteration of the algorithm. (Usually one iteration is sufficient.) We will present the details of our algorithm and discuss its limitations with regard to handling noise and vortices in the unwrapped input phase. The algorithm is robust towards these problems since the Fourier transform is not a pixel-by-pixel operation. We will also discuss the affect of boundary conditions on our phase unwrapping algorithm, as well as a subtle issue involving the continuity of the unwrapped phase.

Figure 1a is an experimental wrapped phase from holography experiments carried out on $\mathrm{Nd}_{2} \mathrm{Fe}_{14} \mathrm{~B}$ hard magnets. Note the presence of vortices (see inset) at the left of the image. We show the unwrapping results of our algorithm in Fig. $1 \mathrm{~b}$ which took 3 iterations and less than 5 sec to complete on a G4 Mac using the scripting language of Gatan's DigitalMicrograph software. The image size was $256 \times 256$ pixels. For comparison, we show the unwrapping results obtained by a pixel-by-pixel, path-dependent method. The path-dependent method, while taking less than a second to complete, incorrectly unwraps the phase near the vortices and propagates the error through the rest of the unwrapped phase. In practice, our algorithm has been found to be quick enough for routine use, exhibiting extremely robust characteristics towards the presence of noise and vortices.

References

1. M. A. Schofield and Y. Zhu, submitted to Phys. Rev. Lett..

2. T. E. Gureyev and K. A. Nugent, J. Opt. Soc. Am. A, 13 (1996) 1670-1682.

3. M. J. Hÿtch, E. Snoeck and R. Kilaas, Ultramicroscopy, 74 (1998) 131-146.

4. V. V. Volkov, Y. Zhu and M. De Graef, Micron, JMIC580 (2002) in press.

5. This work was supported under US DOE contract No. DE-AC02-98CH10886.
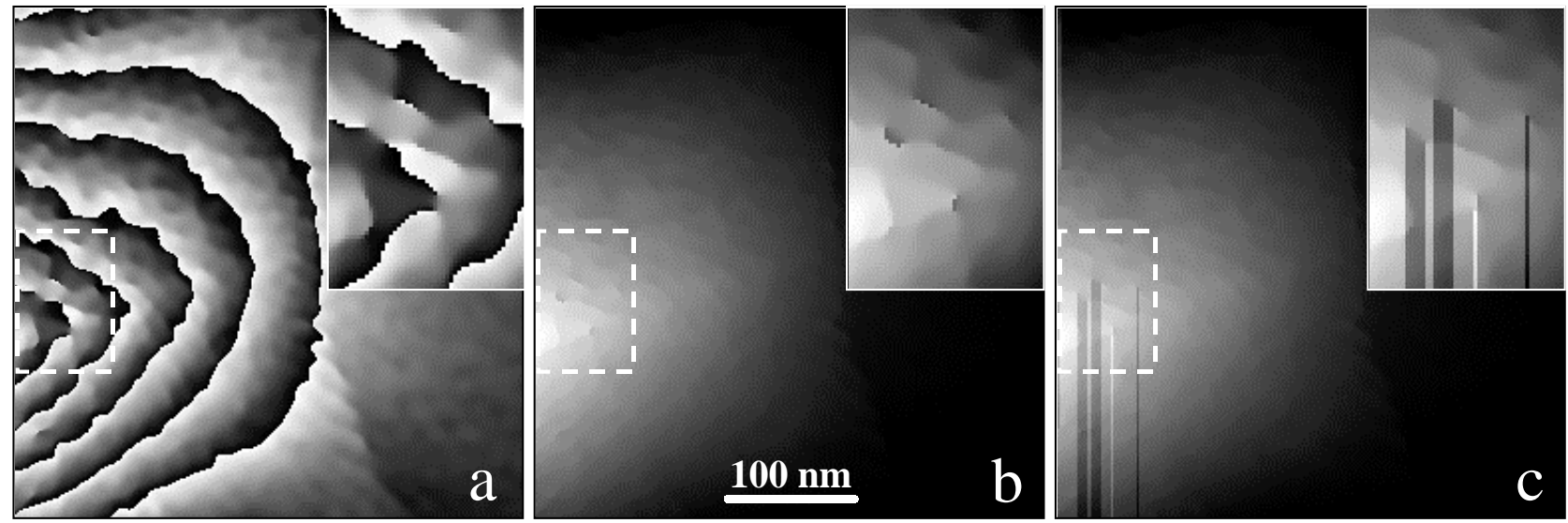

FIG 1. (a) Experimental wrapped phase from holography experiments carried out on $\mathrm{Nd}_{2} \mathrm{Fe}_{14} \mathrm{~B}$ hard magnets. Vortex points (inset) in the phase are present due to low fringe contrast and noise in the original hologram. The unwrapped phase obtained by (b) our algorithm and (c) a path-dependent method. Errors in unwrapping the phase near the vortices present in (a) are not propagated through the solution with our algorithm as shown in the insets. 